\title{
Clustering and Monitoring Edge Behaviour in Enterprise Network Traffic
}

\author{
Christopher Schon \\ Department of Computer Science \\ University College London \\ London, UK \\ Niall Adams \\ Department of Mathematics \\ Imperial College London \\ London, UK
}

Email: christopher.schon.16@ucl.ac.ukEmail:n.adams@imperial.ac.uk

\author{
Marina Evangelou \\ Department of Mathematics \\ Department of Epidemiology and Biostatistics \\ Imperial College London \\ London, UK
}

Email: m.evangelou@imperial.ac.uk

\begin{abstract}
This paper takes an unsupervised learning approach for monitoring edge activity within an enterprise computer network. Using NetFlow records, features are gathered across the active connections (edges) in 15-minute time windows. Then, edges are grouped into clusters using the k-means algorithm. This process is repeated over contiguous windows. A series of informative indicators are derived by examining the relationship of edges with the observed cluster structure. This leads to an intuitive method for monitoring network behaviour and a temporal description of edge behaviour at global and local levels.

Keywords - Cyber-security, clustering, NetFlow.
\end{abstract}

\section{Introduction}

Cyber-security is a growing concern in an increasingly sophisticated technological landscape. Detecting and intercepting an attacker as fast as possible is of utmost importance to prevent companies incurring large costs from lost data or network shutdowns. Traditional, signature-based methods for preventing intrusion are readily circumvented by sophisticated attackers (such as advanced persistent threats [14]), who are well aware of the properties of these defences.

A complementary approach is based on statistical anomaly detection, in which anomalous activity is identified with respect to a statistical model of normal behaviour. Although this area of research is relatively new [1], some recent examples of anomaly detection techniques for enterprise networks have involved quantifying 'normal' activity at a global (entire network) level, such as modelling bursts of traffic [15] or inter-device authentication events [6], and at a local level, such as monitoring specific device connections or clusters [10].

This paper proposes a method that analyses network behaviour by clustering connections between devices in an enterprise network. Specifically, we use NetFlow [3] records, which summarise communications between two devices passing through a router. The objective is to characterise the behaviour of traffic between pairs of devices, that is the behaviour on an edge of the network graph. We intend to detect behaviour that is abnormal from previously observed activity between the devices or abnormal compared to the typical edge behaviour across related subsets of edges or the whole network. In a similar vein to [10], our method identifies anomalous connections and provides a means of locating anomolous subgraphs, and is hence capable of finding malicious network traversals.

First, a mathematical summary in the form of a feature vector is constructed, which describes the behaviour of the edge. Second, these feature vectors are collected into a matrix and presented to a clustering algorithm. Third, comparing feature vectors in the next time window with the present clustering provides a set of derived indicators. Finally, this process iterates over contiguous time windows. In essence, we cluster traffic on the network, rather than network topology characteristics.

For a specific edge, changes in its behaviour can be quantified with respect to the previous window's clustering structure. In this sense, the methodology is designed to detect changes in local traffic behaviour of NetFlow events, and report these changes in an interpretable manner. The methodology presents an intuitive, data-driven monitoring method that complements existing signature-based defence methods. Additionally, the approach provides dashboard-like capabilities to allow monitoring of edge behaviour.

The rest of this paper is structured as follows: Section 2 describes NetFlow records and the large public domain dataset used in our experiments. Section 3 introduces the methodology in two stages, feature construction followed by clustering and derived indicators construction. Section 4 provides examples of monitoring derived indicators on two example edges to demonstrate the situational awareness capabilities of the method.

\section{Data}

NetFlow is a protocol that operates on routers for summarising device connection activity. A NetFlow record contains a variety of information about a connection between devices. We use a recently released cyber-security events dataset from Los Alamos National Laboratory (LANL) [8]. LANL possesses a large enterprise network, which regularly contains thousands of operating devices on any given day. We use a one-week (the first recorded) subset of the data 


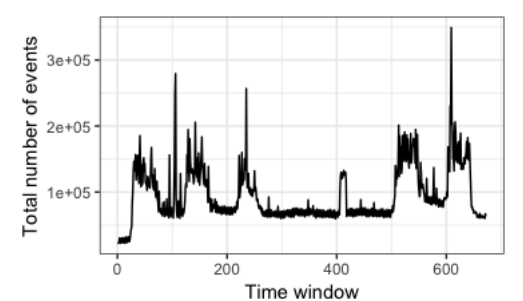

Figure 1: Number of NetFlow events by time window.

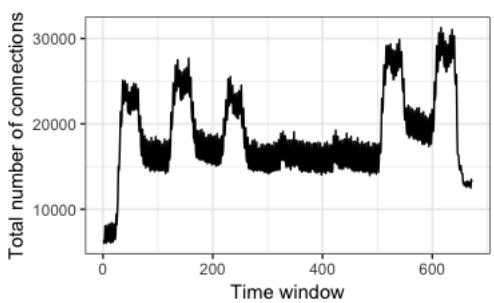

Figure 2: Number of unique connections by time window.

from a 28-day collection period of NetFlow records. This dataset is fully described in [9]. Each record has 9 descriptive fields: time, connection duration, source computer, source port, destination computer, destination port, protocol, packet count and byte count. The first three records of the dataset are presented in 1 . Note that records are collected at second precision with respect to time and connection duration, and that the data has been subjected to various anonymisation procedures. This dataset refers to traffic only 'within' the network.

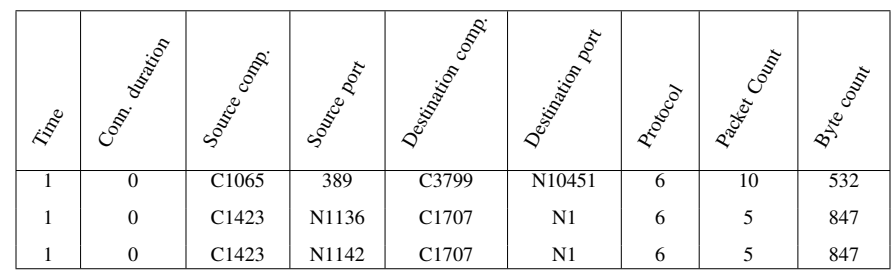

TABLE 1: Example LANL NetFlow records.

Each NetFlow record describes a connection between devices $a$ (source computer) and $b$ (destination computer), starting at time $t$. Often, the direction of the records is meaningful, such that we may speak of a directed connection $a \rightarrow b$, and think about $a$ being the client and $b$ being the server. The record includes the source and destination ports, the latter of which may characterise the type of communication, a count of the number of packets associated with the connection, and the corresponding byte count.

NetFlow records are binned into 15 minute contiguous time windows. This is inclusive on the left but not the right, e.g. the first window contains all events with $0 \leq t<900$. The $i$ th window is denoted by $W_{i}$ and corresponds to the 900 second window starting at time $(i-1) \times 900$. The choice of 15-minute windows is motivated by operational characteristics and published practice. For the former, the rate at which routers dump stored NetFlow records is software configurable. Our experience suggests that this often happens at 5 or 10 minute intervals, thus 15 -minutes is only slightly more than the operational data collection time. For the latter, [11] demonstrated that 15-minute windows provided sufficient granularity to detect sophisticated attack behaviour. For illustration, Figures 1 and 2 show the total number of NetFlow events and the number of unique connections across all time windows.

\section{Methodology}

\subsection{Feature extraction}

Using the mathematical definition from graph theory [12], a graph is a tuple $G=(V, E)$ with $V$ the set of nodes and $E$ the set of edges defining some relationship between pairs of nodes. Here, nodes are the devices of the network and edges represent some relationship between two devices. The edge set $E_{i}$ with cardinality $n_{i}$ will represent the active connections present on the network during $W_{i}$, where active means the occurrence of at least two NetFlow events with one device the client and the other the server. A directed edge between nodes $v_{r}, v_{s} \in V_{i}$ can also be written as $\left(v_{r}, v_{s}\right) \in E_{i}$. The node set for $W_{i}$, denoted $V_{i}$, can then be extracted from the unique elements found in the tuple forms of $e_{i}^{k}, i=1, \ldots, n_{i}$. The reason for the choice of two events will become clear in the construction of the edge features.

Feature vectors that summarise NetFlow records on an edge are constructed. For edge $\left(v_{r}, v_{s}\right)$, each element of the feature vector is a statistic derived from the sequence of field values of the NetFlow entries which have client $v_{r}$ and server $v_{s}$ during $W_{i}$. Let $x_{e_{i}^{k}} \in \mathbb{R}^{16}$ denote the feature vector of edge $e_{i}^{k}$. The elements of this vector are given in Table 2. Let $X_{i} \in \mathbb{R}^{n_{i} \times p}$ denote the feature matrix for $W_{i}$ such that row $j$ corresponds to $x_{e_{i}^{j}}, j=1, \ldots, n_{i}$.

The features in Table 2 are designed to capture average behaviour and variability around this average for different NetFlow fields. As such, attention is restricted to edges with at least two events in the time window.

The last two feature variables, number of events and number of events on modal destination port, characterise the diversity of the types of behaviour on the edge. For example, an edge may only exhibit traffic corresponding to one type of destination port, which corresponds to a specific protocol. In contrast, a number of different protocols could be operating on the edge.

These features lie on different scales, for example, the variance of the number of bytes tends to be much larger than most other features. Each feature component is standardised to have mean and standard deviation 0 and 1 respectively. That is, we construct a standardised edge feature matrix $Z_{i}$ by, over each column of $X_{i}$, subtracting its mean and dividing by its standard deviation. The standardised feature vector for edge $e_{i}^{k}$ is denoted by $z_{e_{i}^{k}}$.

It is worth noting that there are some strong correlations between features. As an example, Figure 3 represents correlations across $W_{59}$. Naturally median and mean measures 
Time related

- Mean, median, standard deviation and inter-quartile range (IQR) of connection duration.

- Mean and standard deviation of inter-arrival times of events.

Bytes related

- Mean, median, standard deviation and IQR of bytes.

Packet related

- Mean, median, standard deviation and IQR of packets.

Other

- Count of the number of events that occured on the edge.

- Count of events on the modal destination port observed on the edge. TABLE 2: NetFlow edge features.

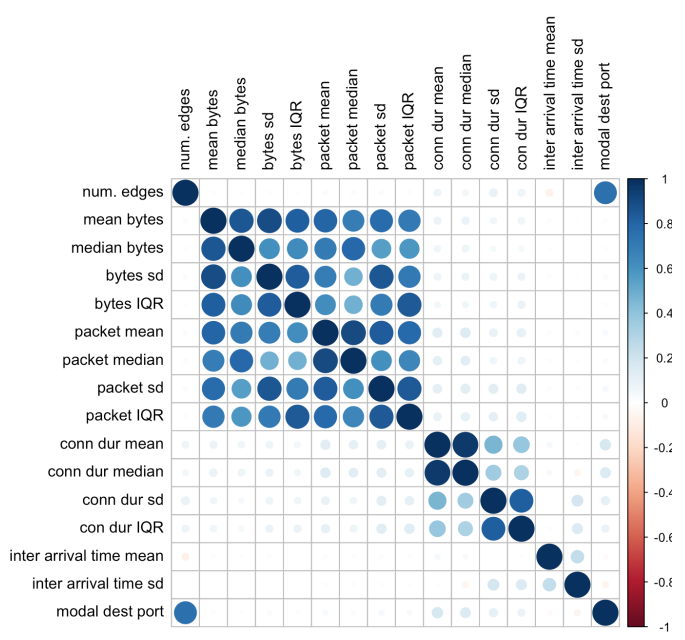

Figure 3: Representation of correlation matrix for $X_{59}$.

are highly correlated, as are the measures of spread (standard deviation and IQR). Most other observed strong correlations lie between packet and bytes related features. This is expected, because the nature of the different protocols tends to produce strong but different relationships between packet and byte related features.

\subsection{Clustering and derived indicators}

We develop a method for clustering edge behaviour across the time windows. There is no response variable in this context, so an unsupervised learning approach is taken. For computational speed, a k-means [5] algorithm is convenient. This is a fast, non-parametric, distance-based method for data clustering. A set of $k$ centroid vectors is iteratively updated to the mean vectors of $k$ sets of closest datapoints to each centroid until some convergence criterion is met. A k-medians algorithm was also tested, but was slower and gave less interpretable centroids vectors. The choice of the number of clusters for either method is not obvious a-priori. To address this, a pilot study using a Gaussian mixture model [4] gives $k=5$ as a sensible choice of parameter.

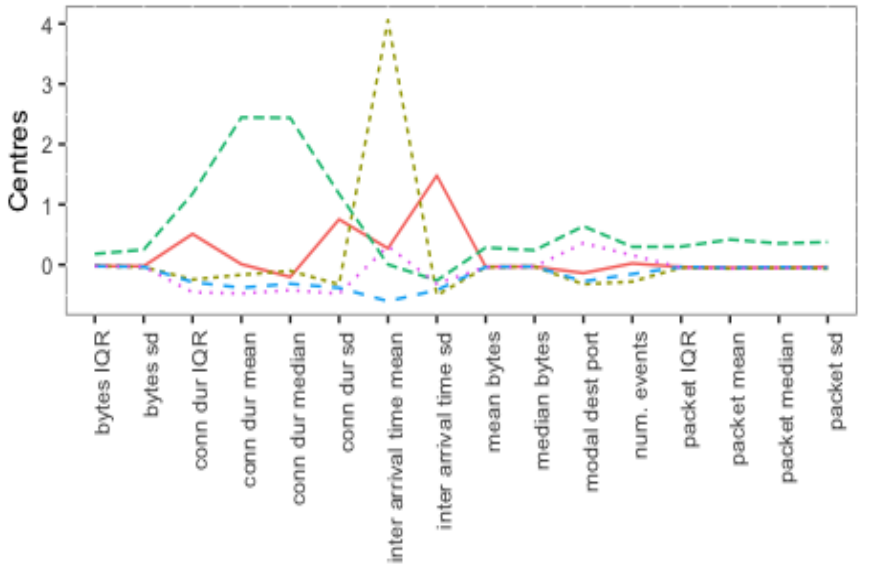

Features

Figure 4: Parallel coordinates plot of k-means centroid vectors for $Z_{59}$.

For illustration, Figure 4 displays a parallel coordinates plot of the centroid vectors derived by clustering of $Z_{59}$. This figure demonstrates several different types of edge behaviour during $W_{59}$ in the network. For example, the cluster associated with the lighter green line is notable since it is characterised by larger connection duration mean and connection duration median of NetFlow events.

Next, we build a sequential method of constructing informative statistics intended for further human or statistical analysis. K-means clustering with 5 centroid vectors is conducted using standardised edge features over each of the chosen time windows. Let $M_{i}=\left\{C_{i}, \boldsymbol{a}_{i}\right\}$ represent the k-means clustering for $W_{i}$, where $C_{i} \in \mathbb{R}^{16 \times 5}$ is the matrix containing the 5 centroid vectors, and $\boldsymbol{a}_{i}$ is a vector of length $n_{i}$ where the $k$ th element corresponds to the assigned cluster label of $e_{i}^{k}$.

We wish to monitor edges by capturing specific aspects of edge behaviour with respect to the clustering in the previous time window. Specifically for each edge $e_{i}^{k}, k=1, \ldots, n_{i}$, we compute:

1) An indicator variable for $e_{i}^{k} \notin E_{i-1}$. This captures whether or not the edge was inactive in the previous time window. If this is 1 , the edge is new.

$$
I_{i}^{e_{i}^{k}, 1}= \begin{cases}1, & \text { if } e_{i}^{k} \notin E_{i-1} . \\ 0, & \text { otherwise. }\end{cases}
$$

2) An indicator variable for outlier edges. For the definition of an outlier in this context, we take the empirical cumulative distribution function (ecdf) of the Euclidean distances of all $e_{i-1}^{k} \in E$ from their corresponding assigned cluster centroid $C_{i-1}^{\boldsymbol{a}_{i-1}^{k}}$. Let this distance be denoted $d_{i}^{k}$ and for $i>1$ let $M_{i-1}$ 's ecdf function for such distances be $\hat{F}_{M_{i-1}}(d)$.

$$
I_{i}^{e_{i}^{k}, 2}= \begin{cases}1, & \text { if } \hat{F}_{M_{i-1}}\left(d_{i}^{k}\right)>0.99 . \\ 0, & \text { otherwise. }\end{cases}
$$




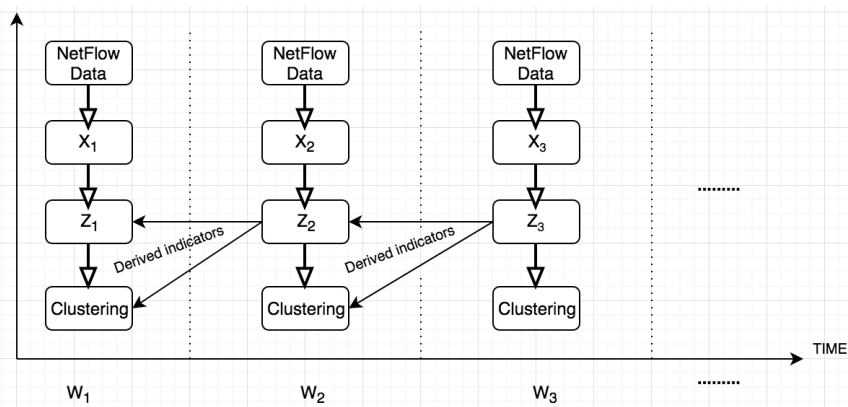

Figure 5: Illustration of process for construction of derived indicator variables.

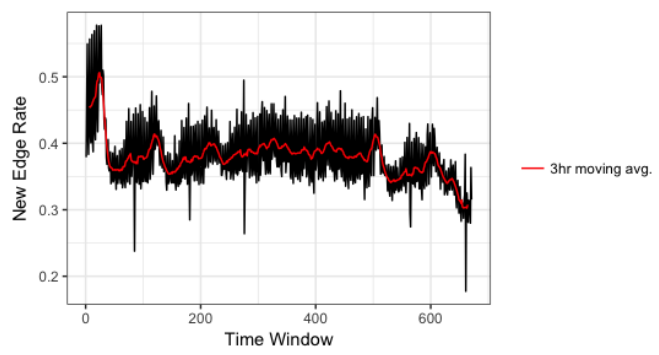

Figure 6: New edge rates $\frac{1}{n_{i}} \sum_{k=1}^{n_{i}} I_{i}^{e_{i}^{k}, 1}$

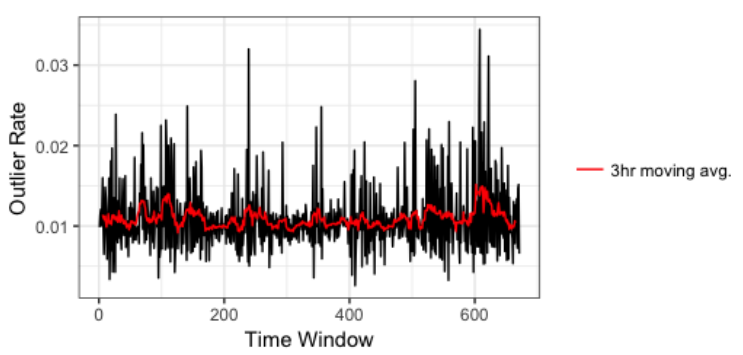

Figure 7: Outlier rates $\frac{1}{n_{i}} \sum_{k=1}^{n_{i}} I_{i}^{e_{i}^{k}, 2}$.

3) A variable indicating whether the cluster assignment of an edge has changed from the previous window. Define $l$, where appropriate, as the index of $\boldsymbol{a}_{i-1}$ corresponding to edge $e_{i}^{k}$.

$$
I_{i}^{e_{i}^{k}, 3}= \begin{cases}1, & \text { if } I_{i}^{e_{i}^{k}, 1}=0 \text { and } \min _{j}\left\|z_{e_{i}^{k}}-C_{i-1}^{j}\right\| \neq \boldsymbol{a}_{i-1}^{l} \\ 0, & \text { otherwise. }\end{cases}
$$

In the case where $e_{i}^{k} \notin E_{j}$, we set $I_{j}^{e_{i}^{k}, l}=0, l=1,2,3$.

From these, sequences of informative binary indicators are derived across the time windows. The steps of this process are illustrated in Figure 5.

Averaging these indicators over the edge sets and comparing with 3 hour moving averages yields Figures 6-8. These figures show that new edge rates and outlier rates remain relatively constant, and cluster change rates show some seasonality. Exploratory analysis of the data suggests the records start on a Wednesday. Hence, new cluster rates show more variability during weekdays, as the rate is visibly

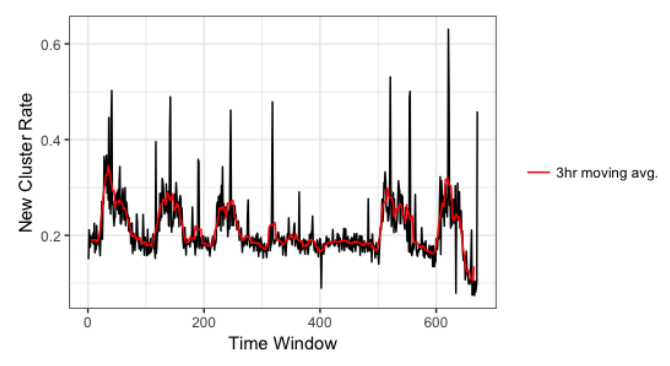

Figure 8: Cluster change rates $\frac{1}{n_{i}} \sum_{k=1}^{n_{i}} I_{i}^{e_{i}^{k}, 3}$.

flatter between time windows 300 and 500, that is between the early hours of Saturday and Monday morning.

The indicators give a motivation for constructing control charts [13], as a way of monitoring the indicator process over time. It is rare for an edge to be persistently active across the week, so averaging needs to be done over the number of windows for which the edge is active, not the total observation period. We introduce further notation for the presence of edge $e_{i}^{k}$ in other time windows:

$$
T_{e_{i}^{k}}^{j}= \begin{cases}1, & \text { if } e_{i}^{k} \in E_{j} \\ 0, & \text { otherwise. }\end{cases}
$$

A control chart for edge $e_{i}^{k}$ for each indicator can be derived over a period of time by averaging, at each time $t$, the indicator values starting from time $t_{0}$, the first occurrence of the edge. Let $m_{e_{i}^{k}}^{t}=\sum_{j=t_{0}}^{t} T_{e_{i}^{k}}^{j}$, i.e. the number of windows edge $e_{i}^{k}$ was active between time windows $t_{0}$ and $t$. If we assume the sequence of indicators is a sequence of independent and identically distributed Bernoulli trials, with parameter $p_{e_{i}^{k}}^{t, l}$, then the maximum likelihood estimator for the $l$ th indicator is:

$$
\hat{p}_{e_{t}^{k}}^{t, l}=\frac{1}{m_{e_{i}^{k}}^{t}} \sum_{j=t_{0}}^{t} I_{j}^{e_{i}^{k}, l}
$$

with standard deviation:

$$
\operatorname{sd}\left(\hat{p}_{e_{i}^{k}}^{t, l}\right)=\sqrt{\frac{\hat{p}_{e_{i}^{k}}^{t, l}\left(1-\hat{p}_{e_{i}^{k}}^{t, l}\right)}{m_{e_{i}^{k}}^{t}}}
$$

We construct a simple, approximate control chart by estimating the in-control parameters over the one day following the edge's first appearance in the edge set. The upper and lower control limits of the chart are fixed to \pm 1.96 standard deviations from the maximum likelihood estimate, Equation (5). The estimate is then updated sequentially and anomalies are flagged when the estimate first crosses the control limits.

\section{Examples}

We now illustrate the monitoring potential of the proposed methodology, by considering two example edges in the network, denoted $e_{1}$ and $e_{2}$. Note that a sequential 


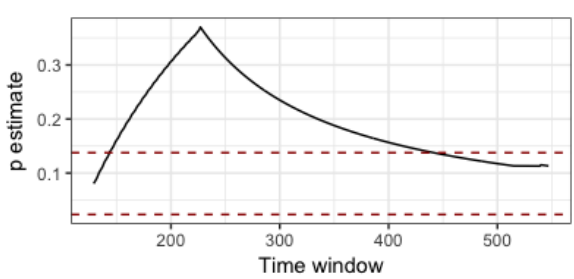

Figure 9: $\hat{p}_{e_{1}}^{t, 1}$.

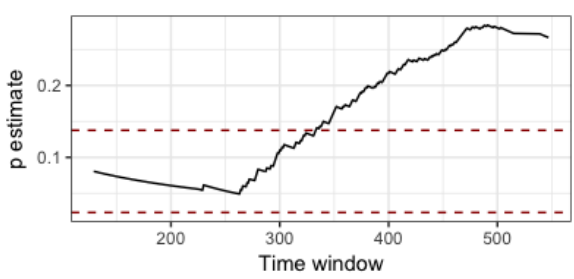

Figure 10: $\hat{p}_{e_{1}^{t}}^{t, 2}$.

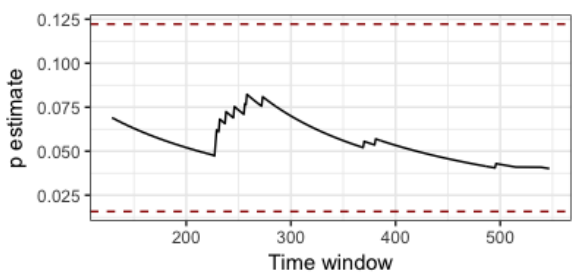

Figure 11: $\hat{p}_{e_{1}}^{t, 3}$.

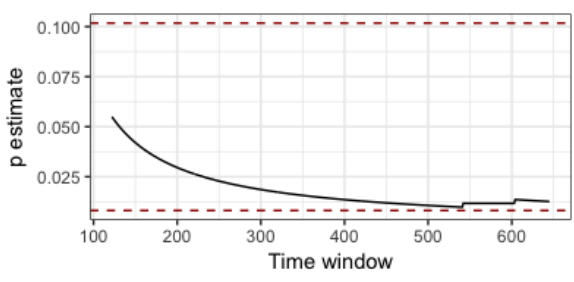

Figure 12: $\hat{p}_{e_{2}}^{t, 1}$.

control chart would flag an exit from the control limits, after which a new burn-in is required to estimate new in-control parameters. Here, the full pictures are given for illustration.

Figure 9 shows that $\hat{p}_{e_{1}}^{t, 1}$ exited its control limit at around $W_{150}$. Figures 10 and 11 show that the edge began to observe a higher rate of outlier features in the second half of the observation period, but the cluster change rate remained constant.

Figures 12-14 show $e_{2}$ 's control charts. In this case, cluster change and new edge rates remained within the control limits throughout the entire observation period, whereas the outlier rate fell below its lower limit at around $W_{280}$.

Another use of our methodology is the thinning of the data to edges which exhibit 'unusual' behaviour according to their indicator function values. This is because each indicator value characterises edge behaviour in relation to the behaviour in the previous time window, with a value of 1 expressing a departure from the previous behaviour. Studying locally anonymous subgraphs of enterprise networks has

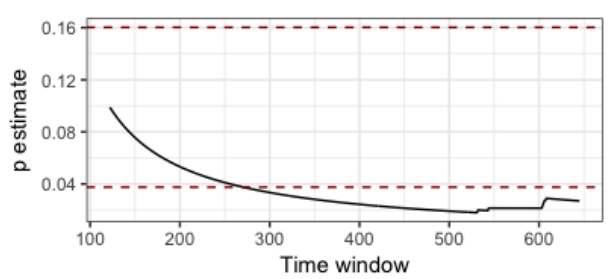

Figure 13: $\hat{p}_{e_{2}}^{t, 2}$.

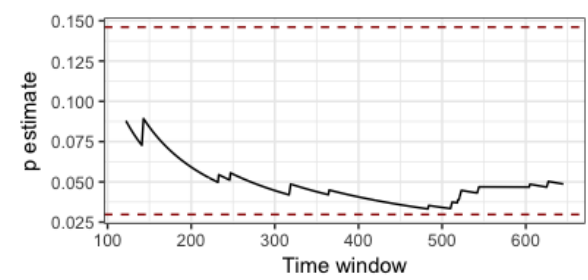

Figure 14: $\hat{p}_{e_{2}}^{t, 3}$.

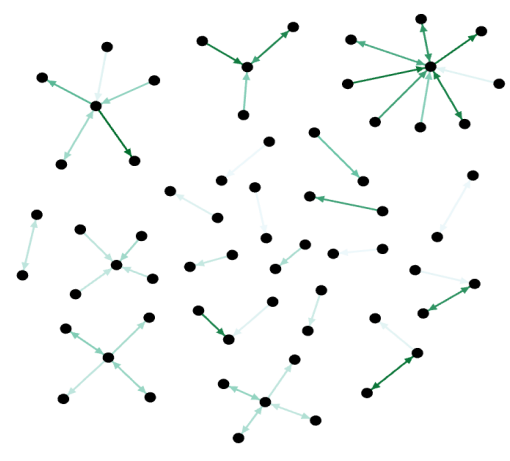

Figure 15: $F_{58}$, with connection duration mean colour scaling.

proven to be an effective approach for detecting anomalous activity [10]. Using our methodology, one definition of anomalous edges in time window $i$ is the reduced edge set $F_{i}=\left\{e_{i}^{k} \in E_{i}: \sum_{l} I_{i}^{e_{i}^{k}, l}=2\right\}$. This corresponds to edges that are both an outlier and are either a new edge or see a change in cluster assignment. So two types of behavioural change are observed on each edge of $F_{i}$. For $W_{59}$ this gives a total of 47 edges, reduced from an original set of 15464 .

This allows for visual interpretation of edge behaviour that is not easy on the entire network graph due to the volume of nodes and edges. Figure 15 displays the network graph for the edge set $F_{58}$, where colouring relates to the edge connection duration mean feature.

Some structure is present in Figure 15, such as the presence of a few nodes which are source and/or destination to several anomalous edges. It could be that outlier edges to/from this node are not uncommon. This can easily be checked using the control chart method.

Figure 17 shows the same network graph, where edges are now coloured by cluster assignment, the centroids of which are given in Figure 16. A portion of anomalous edge activity are assigned to the purple cluster. These are edges 


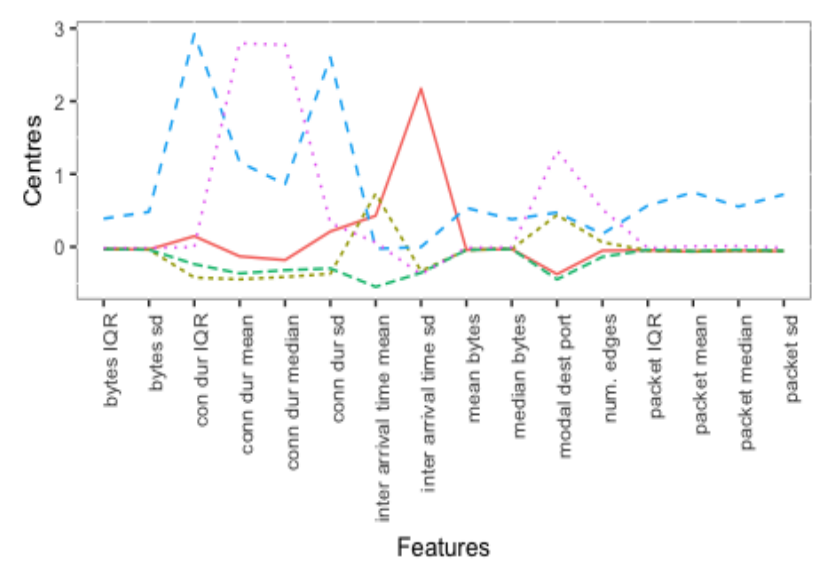

Figure 16: Parallel coordinates plot for cluster centroids of $Z_{58}$.

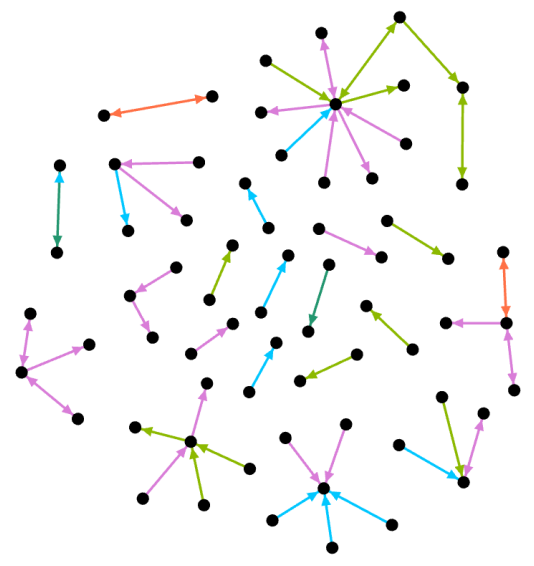

Figure 17: $F_{58}$ topology with edges coloured by cluster assignment corresponding to centroids in Figure 16.

with larger connection duration mean, connection duration median, num. events and modal destination port values.

\section{Conclusion}

Network monitoring and anomaly detection for cybersecurity purposes are challenging because of the data rates and volumes involved. However, such monitoring provides the promise of extra support tools for situational awareness and network cognisance. The method described, while simple, appears to be capable of dealing with the rate of data collection observed for the LANL network data. It allows us to create a descriptive summary of the network, which captures edge behaviour rather than network topology. We can construct different scales of data analysis: individual edges, specific subgraphs, and entire network edge-behaviour. This provides a foundation for a complementary enterprise network analysis tool for detecting abnormal edge behaviour.

\section{Acknowledgments}

This work was supported by a UK Engineering and Physical Sciences Research Council (EPSRC) studentship.
Figures were produced by graph analysis software Gephi [2] and R [7] library ggplot2 [16].

\section{References}

[1] N. Adams and N. Heard. Dynamic Networks and Cyber-security, volume 1. World Scientific, 2016.

[2] M. Bastian, S. Heymann, M. Jacomy, et al. Gephi: an open source software for exploring and manipulating networks. In International Conference on Web and Social Media, volume 8, pages 361-362. ICWSM, 2009.

[3] I. Cisco. Netflow-Cisco systems. URL: http://www. cisco. com/go/netflow, 2006.

[4] C. Fraley and A. E. Raftery. Enhanced modelbased clustering, density estimation, and discriminant analysis software: Mclust. Journal of Classification, 20(2):263-286, 2003.

[5] J. A. Hartigan and M. A. Wong. Algorithm AS 136: A k-means clustering algorithm. Journal of the Royal Statistical Society. Series C (Applied Statistics), 28(1):100-108, 1979.

[6] N. Heard and P. Rubin-Delanchy. Network-wide anomaly detection via the Dirichlet process. In Intelligence and Security Informatics (ISI), 2016 IEEE Conference, pages 220-224. IEEE, 2016.

[7] R. Ihaka and R. Gentleman. R: a language for data analysis and graphics. Journal of Computational and Graphical Statistics, 5(3):299-314, 1996.

[8] A. D. Kent. Comprehensive, multisource cyber-security data set. URL: http://dx.doi.org/10.17021/1179829, 2015.

[9] A. D. Kent. Cyber security data sources for dynamic network research. Dynamic Networks and CyberSecurity, pages 51-53, 2016.

[10] J. Neil, C. Hash, A. Brugh, M. Fisk, and C. B. Storlie. Scan statistics for the online detection of locally anomalous subgraphs. Technometrics, 55(4):403-414, 2013.

[11] J. Neil, C. Storlie, C. Hash, and A. Brugh. Statistical detection of intruders within computer networks using scan statistics. In Data Analysis for Network CyberSecurity, pages 71-104. World Scientific, 2014.

[12] O. Ore and Y. Ore. Theory of Graphs, volume 38. American Mathematical Society, Providence, RI, 1962.

[13] S. Roberts. A comparison of some control chart procedures. Technometrics, 8(3):411-430, 1966.

[14] C. Tankard. Advanced persistent threats and how to monitor and deter them. Network Security, 2011(8):16-19, 2011.

[15] A. Valdes and K. Skinner. Adaptive, model-based monitoring for cyber attack detection. In International Workshop on Recent Advances in Intrusion Detection, pages 80-93. Springer, 2000.

[16] H. Wickham. GGPLOT2: Elegant Graphics for Data Analysis. Springer, 2016. 